\title{
FARELO DA RAIZ INTEGRAL DE MANDIOCA EM DIETAS DE FRANGOS DE CORTE TIPO CAIPIRA
}

\author{
CASSAVA ROOT MEAL IN DIETS OF FREE-RANGE BROILER CHICKENS
}

\author{
Souza, K.M.R. ${ }^{1 *}$, Carrijo, A.S. ${ }^{1 \mathrm{~A}}$, Kiefer, C. ${ }^{1 \mathrm{~B}}$, Fascina, V.B. ${ }^{1 \mathrm{C}}$, Falco, A.L. ${ }^{1 \mathrm{D}}$, Manvailer, G.V. ${ }^{1 \mathrm{E}}$ \\ e García, A.M.L. ${ }^{1 F}$
}

${ }^{1}$ FAMEZ/UFMS. Universidade Federal de Mato Grosso do Sul. Av. Senador Filinto Muller. Campo Grande, MS. Brasil. *karinamrs@gmail.com; Aacarrijo@nin.ufms.br; ${ }^{B}$ charles@nin.ufms.br; cvitorfascina@yahoo.com.br; Ddry_f@hotmail.com; Egvmanvailer@hotmail.com; Fameliagarc@yahoo.com.br

\section{PalaVRas ChaVes adicionais}

Cor de pele. Ganho de peso. Pescoço pelado. Propriedades funcionais da carne. Rendimento de carcaça. Sistema de criação.

\section{RESUMO}

O objetivo do trabalho foi avaliar níveis de inclusão de farelo da raiz integral de mandioca, em dietas de frangos de corte tipo caipira, sobre o desempenho, os rendimentos de carcaça, de cortes e as propriedades funcionais da carne. Foram distribuídos 384 pintainhos machos da linhagem ISA S757-N (pescoço pelado) em um delineamento inteiramente casualizado com quatro tratamentos e quatro repetições de 24 aves cada. Os tratamentos foram $0 \%, 20 \%, 40 \%$ e $60 \%$ de FRIM (farelo da raiz integral de mandioca) na ração total. O período experimental foi dividido em três fases de criação: inicial ( 1 a 28 dias de idade), crescimento ( 29 a 56 dias de idade) e engorda (57 a 84 dias de idade). Foram obtidos o peso corporal final (PF), o ganho de peso (GP), consumo de ração, conversão alimentar e viabilidade. Aos 84 dias foram obtidos os rendimentos de carcaça, peito, coxa+sobrecoxa, asa, dorso gordura abdominal e das carnes de peito das aves foram determinados $\mathrm{o} \mathrm{pH}$, capacidade de retenção de água (CRA) e perda de peso por cozimento (PPC). Por comparação visual, foram determinados os escores para as cores de pele de canela e de peito. Os resultados foram submetidos à análise de variância, à análise de regressão e as médias

\#Parte da dissertação do primeiro autor.

\section{Additional KEYWORdS}

Color of skin. Meat functional properties. Naked neck. Rearing system. Weight gain. Yield of carcass.

foram comparadas pelo teste de Tukey a $5 \%$ de probabilidade. A análise de variância detectou diferença significativa entre os tratamentos para PF e GP, somente na fase de 1 a 28 dias de idade. Foi verificado aumento linear para PF e GP à medida que se aumentou o nível de inclusão de FRIM na ração. Não existiram diferenças significativas entre os tratamentos para as variáveis de rendimento de carcaça, de cortes, $\mathrm{pH}, \mathrm{CRA}$ e PPC. A utilização do FRIM nas dietas de frangos de corte tipo caipira promoveu redução linear na coloração das peles da canela e do peito das aves com o aumento do nível de inclusão desse ingrediente nas rações. O farelo da raiz integral de mandioca pode ser utilizado nas dietas de frangos de corte tipo caipira na proporção de $60 \%$ da ração total sem prejudicar o desempenho, os rendimentos de carcaça, de corte e as propriedades funcionais da carne, no entanto promove descoloração das peles de canela e peito.

\section{SUMMARY}

The aim this work was to evaluate cassava root meal inclusion levels in diets of free-range broiler chickens on performance, carcass yield, parts yield and functional properties of the meat. 384 male chickens of ISA S757-N strain (naked 
neck) were distributed in a completely randomized design with four treatment and four replicates of 24 birds each. The treatments were 0\%, 20\%, $40 \%$ and $60 \%$ of FRIM (cassava root meal) in the total ration. The trial period was divided into three rearing phases: initial ( 1 to 28 days of age), growth (29 to 56 days of age) and fattening ( 57 to 84 days of age). Final body weight (PF), weight gain (WG), feed intake, feed conversion and liveability were evaluated. At $84^{\text {th }}$ day, carcass, breast, thigh + upper thigh, wing, back and abdominal fat yield were obtained. Breast meat was used to determine the $\mathrm{pH}$, water holding capacity (CRA) and cooking loss (PPC) too. The shank and breast skin color scores were determined by visual comparison. The results were submitted to analysis of variance, analysis of regression and averages were compared by Tukey's test to $5 \%$ of probability. The analysis of variance found a significant difference between treatments for BW and WG, only at the phase of 1 to 28 days of age. There was a linear increased in BW and WG when the CRM inclusion level increased in feed. There were no significant differences between the treatments for the variables of carcass yield, cuts yield, $\mathrm{pH}$, CRA and PPC. The use of FRIM in the diets of freerange broiler chickens promoted linear reduction in the shank and breast skin colors with the increase of the inclusion level of this ingredient in the feed. The FRIM can be used in the diets of freerange broiler chickens in the proportion of $60 \%$ of total ration without resulting in worst performance, carcass yield, cuts yield and functional properties of the meat, however it promotes shank and breast skin no coloration.

\section{INTRODUÇÃO}

Os consumidores, preocupados com a segurança dos alimentos e em busca de uma nutrição mais próxima do natural, proporcionaram o aumento da demanda por produtos avícolas com maiores atributos de qualidade. Essa preocupação influenciou o sistema de produção na avicultura, sendo que, a criação de frangos de corte de crescimento lento no sistema semiintensivo ou caipira tem sido uma alternativa apropriada para satisfazer o mercado.

O frango tipo caipira permite algumas adaptações no sistema de criação, tendo em vista a grande rusticidade e resistência das aves em relação ao frango de granja de escala industrial. $\mathrm{O}$ aspecto mais marcante associado a esse sistema é o fato das aves terem acesso a piquetes com área verde para o exercício diário, o que não prejudica o desempenho dos frangos (Takahashi et al., 2006).

No entanto, mesmo no sistema de produção tipo caipira, a alimentação representa cerca de $70 \%$ do custo da atividade (UBA, 2007) e ingredientes alternativos, como a mandioca, podem ser utilizados para tornar as rações menos onerosas. Vários estudos foram feitos para avaliar a substituição de cereais por farelo de mandioca em dietas de aves e os resultados variaram em relação aos valores alimentares, problemas nutricionais, e desempenho produtivo (Enriquez e Ross, 1967; Garcia e Dale, 1999; Carrijo et al., 2002). A inclusão do farelo de mandioca, em rações para frangos de corte, pode ser de até 55\% sem prejudicar o desempenho das aves, desde que a dieta contenha níveis adequados de metionina para o correto balanceamento da ração (Carrijo et al., 2002).

Além disso, a indústria vem mostrando maiores preocupações com o rendimento das carcaças e dos cortes, devido às exigências dos consumidores por peças que apresentem boa conformação, menor peso e quantidade de gordura na carcaça (Hellmeister Filho, 2002). Entretanto, existe a busca não somente pela satisfação do valor nutricional, mas também pelo prazer com o ato de comer.

Dessa forma, o objetivo do trabalho foi avaliar níveis de inclusão de farelo da raiz integral de mandioca, em dietas de frangos de corte tipo caipira, sobre o desempenho, rendimentos de carcaça, de cortes e as propriedades funcionais da carne.

\section{MATERIAL E MÉTODOS}

O experimento foi realizado no laboratório experimental em ciência aviária da Faculda- 


\section{MANDIOCA PARA FRANGOS DE CORTE TIPO CAIPIRA}

de de Medicina Veterinária e Zootecnia da Universidade Federal de Mato Grosso do Sul, no período de 14 de agosto a 06 de novembro de 2007.

Foram distribuídos 384 pintainhos machos da linhagem ISA S757-N (pescoço pelado) em delineamento inteiramente casualizado com quatro tratamentos e quatro repetições de 24 aves cada. Os tratamentos foram níveis de 0, 20, 40 e 60\% de FRIM (farelo da raiz integral de mandioca) na ração total, ocorrendo respectivamente a $0 ; 28 ; 48 ; 56 ; 96$ e 85,44\% de substituição da quantidade milho em relação à ração do tratamento controle que foi composta basicamente de milho e farelo de soja.

O período experimental foi dividido em três fases de criação: inicial ( 1 a 28 dias de idade), crescimento (29 a 56 dias de idade) e engorda ( 57 a 84 dias de idade). As dietas apresentaram a mesma composição nutricional nos períodos experimentais. As rações foram fareladas, isoenergéticas, isoprotéicas e balanceadas a fim de atender as exigências nutricionais conforme recomendações de Rostagno et al. (2005) para aves de reposição semipesadas, exceto para o nível de proteína bruta (PB), sendo utilizado o valor de $15,3 \%$, no entanto as exigências sugeridas para os aminoácidos limitantes foram supridas (tabela I).

Cada box foi equipado com uma campânula que continha duas lâmpadas incandescentes de $100 \mathrm{~W}$ para o aquecimento, um comedouro tubular e um bebedouro pendular. A cama utilizada foi de casca de estilosantes com oito centímetros de espessura. As aves permaneceram confinadas até os 28 dias de idade e a partir do $29^{\circ}$ dia tiveram livre acesso ao piquete de gramíneas. O programa de luz adotado foi o de iluminação artificial de 24 horas nos primeiros 14 dias e de luz natural até o final do período experimental. Diariamente foram verificadas e anotadas as temperaturas (máxima e mínima) e a ocorrência de mortalidade.

As aves e as rações foram pesadas no dia do alojamento, aos 28, 56 e 84 dias de idade. As variáveis analisadas foram: peso corporal final (PF), ganho de peso (GP), consumo de ração (CR), conversão alimen$\operatorname{tar}$ (CA) e viabilidade (VB). GP e CA foram corrigidos pela mortalidade segundo Sakomura e Rostagno (2007). Com o objetivo de iniciar as avaliações da fase conseguinte, aos 28 e 56 dias, as aves foram redistribuídas para homogeneização dos pesos das repetições.

O PF foi o peso absoluto médio das aves no final de cada fase. O GP foi obtido pela diferença entre PF e peso inicial. A partir da subtração da quantidade de ração fornecida e das sobras, sendo o resultado dividido pelo número de aves e posteriormente pelo número de dias de cada período (28 dias), foi determinado o CR.

Os resultados de CA foram encontrados pela relação entre CR e GP. A VB foi obtida pela fórmula:

$$
V B=[1-(M O R T / A A) \times 100]
$$

onde:

$\mathrm{VB}=$ viabilidade .

MORT = mortalidade ocorrida no período.

$\mathrm{AA}=$ número de aves no alojamento.

Aos 84 dias de idade foram retiradas duas aves por repetição com peso corporal correspondente ao peso corporal médio da unidade experimental $\pm 5 \%$ que foram identificadas por anilhas numeradas em uma das patas. As aves foram submetidas a jejum alimentar por 8 horas e pesadas antes do abate, que foi realizado por sangria, após desensibilização.

No momento da evisceração foram retiradas a gordura presente na região próxima a cloaca e aquela aderida à moela (gordura abdominal). As carcaças sem pés, cabeça e pescoço foram pesadas. Posteriormente foram feitos os cortes de peito, coxa+ sobrecoxa, asa e dorso. O rendimento de carcaça (RCAR) foi encontrado a partir da relação entre o peso absoluto da carcaça e o 
Tabela I. Composição percentual e calculada das dietas experimentais. (Composition and calculated percentage of the experimental diets).

\begin{tabular}{|c|c|c|c|c|}
\hline \multirow[t]{2}{*}{ Ingredientes (\%) } & \multicolumn{4}{|c|}{ Níveis de inclusão de FRIM } \\
\hline & $0 \%$ & $20 \%$ & $40 \%$ & $60 \%$ \\
\hline Milho & 70,23 & 51,70 & 29,16 & 3,80 \\
\hline FRIM & - & 20,00 & 40,00 & 60,00 \\
\hline Farelo de soja & 18,49 & 21,49 & 25,66 & 28,77 \\
\hline Farelo de trigo & 5,00 & 2,96 & - & - \\
\hline Óleo de soja & - & - & 1,47 & 3,56 \\
\hline Fosfato bicálcico & 1,75 & 1,81 & 1,88 & 1,93 \\
\hline Calcário & 1,13 & 0,99 & 0,84 & 0,71 \\
\hline Sal & 0,41 & 0,40 & 0,40 & 0,39 \\
\hline DL-Metionina & 0,15 & 0,19 & 0,21 & 0,26 \\
\hline L-Lisina HCL & 0,26 & 0,20 & 0,12 & 0,20 \\
\hline Treonina & 0,07 & 0,07 & 0,06 & 0,18 \\
\hline Premix vitamínico ${ }^{1}$ & 0,10 & 0,10 & 0,10 & 0,10 \\
\hline Premix mineral $^{2}$ & 0,10 & 0,10 & 0,10 & 0,10 \\
\hline Caulim & 2,31 & - & - & - \\
\hline Total & 100,00 & 100,00 & 100,00 & 100,00 \\
\hline \multicolumn{5}{|l|}{ Valores calculados } \\
\hline Energia metabolizável (kcal/kg) & 2.900 & 2.900 & 2.900 & 2.900 \\
\hline Proteína bruta (\%) & 15,30 & 15,30 & 15,30 & 15,30 \\
\hline Fibra bruta (\%) & 2,70 & 3,43 & 4,06 & 4,87 \\
\hline Metionina digestível (\%) & 0,38 & 0,40 & 0,42 & 0,45 \\
\hline Metionina+cistina digestível (\%) & 0,61 & 0,62 & 0,62 & 0,62 \\
\hline Lisina digestível (\%) & 0,85 & 0,85 & 0,85 & 0,95 \\
\hline Treonina digestível (\%) & 0,57 & 0,57 & 0,57 & 0,67 \\
\hline Sódio (\%) & 0,18 & 0,18 & 0,18 & 0,18 \\
\hline Cálcio (\%) & 0,94 & 0,94 & 0,94 & 0,94 \\
\hline Fósforo disponível (\%) & 0,43 & 0,43 & 0,43 & 0,43 \\
\hline
\end{tabular}

${ }^{1}$ Quantidade/kg: vit. $\mathrm{B}_{6} 2500 \mathrm{mg}$; vit. $\mathrm{B}_{12}: 14000 \mathrm{mcg}$; niacina: $35000 \mathrm{mg}$; acido fólico: $1000 \mathrm{mg}$; acido pantotênico: 12000 mg; biotina: 80 mg; vit. A: 10500000 Ul; vit. $D_{3:} 2500000$ Ul; vit. E: 20000 Ul; vit. K 2500 mg; vit. $B_{1:} 2000$ mg; vit. B 5000 mg; bht: 100 mg; ${ }^{2}$ quantidade/kg: Zn 110000 mg; Se: 360 mg; l: 1400 mg; Cu: 20000 mg; Mn: 156000 mg; Fe: 96000 mg.

peso corporal antes do abate. Os rendimentos de peito (RPTO), coxa+sobrecoxa (RCSX), asa(RASA), dorso(RDOR), cabeça+ pescoço (RCPC) e de gordura abdominal (RGAB) foram determinados em relação ao peso da carcaça eviscerada sem pés, cabeça e pescoço.

As análises de capacidade de retenção de água (CRA) e perda de peso por cozimento (PPC) foram determinadas 24 horas post mortem utilizando-se a carne de peito de uma das aves. A CRA foi efetuada em duplicata e para cada réplica foram utilizados dois gramas de amostra de carne de peito que foram colocados entre duas folhas de papel filtro circular e esse conjunto entre duas placas de vidro que receberam um peso de $10 \mathrm{~kg}$ durante cinco minutos. As amostras foram pesadas após a pressão e por diferença calculou-se a quantidade de água perdida. O resultado foi expresso em porcentual de água exsudada em relação ao

Archivos de zootecnia vol. 60, núm. 231, p. 492. 


\section{MANDIOCA PARA FRANGOS DE CORTE TIPO CAIPIRA}

peso inicial da amostra.

Para a PPC, amostras de filés íntegros foram pesadas em duplicata, embaladas em papel laminado e cozidas em chapa metálica pré-aquecida e regulada para $180^{\circ} \mathrm{C}$, permanecendo por quatro minutos de cada lado. Após o cozimento, os filés foram retirados do papel laminado e resfriados até \pm $40^{\circ} \mathrm{C}$ sobre papel absorvente em temperatura ambiente. Os filés cozidos foram pesados para que a PPC fosse determinada através da subtração do peso inicial (carne in natura) e final (carne cozida) e o resultado obtido dividido pelo peso inicial.

A carne de peito da segunda ave foi utilizada para a determinação do pH que foi realizada aos 45 minutos post mortem. Os peitos foram dessecados e as carnes foram moídas em processador tipo mix. A análise foi feita em duplicata e para cada réplica foram utilizados dois gramas de carne. Cada amostra foi colocada em béquer onde foi adicionado $20 \mathrm{ml}$ de água destilada. A solução foi agitada por dois minutos e posteriormente realizou-se a leitura do $\mathrm{pH}$ pela inserção do eletrodo no béquer que continha a solução.

As cores da pele de canela (CPCN) e a da pele de peito (CPPT) foram obtidas por comparação visual com o auxílio do leque DSM para cor de pele de frango o qual contém atribuição de cores que variam de 101 a 108, equivalendo respectivamente a cor mais branca e a cor mais amarelada. Foi atribuído o valor 100 quando se observou uma cor mais branca que a do leque de comparação.

Os resultados foram submetidos à análise de variância, posteriormente à análise de regressão com o auxílio do programa ESTAT e quando ocorreram diferenças significativas, as médias foram comparadas pelo teste de Tukey HSD a 5\% de probabilidade.

\section{RESULTADOS}

As temperaturas máxima e mínima medidas durante o período de criação de 1 a 84
Tabela II. Desempenho de frangos de corte tipo caipira, em três fases de criação, submetidos a dietas contendo farelo de raiz integral de mandioca (FRIM). (Free range broiler chickens performance in three phases, fed diets containing cassava root meal (FRIM)).

\begin{tabular}{|c|c|c|c|c|c|}
\hline \multirow[b]{2}{*}{ Variáveis } & \multicolumn{5}{|c|}{ Níveis de FRIM } \\
\hline & $0 \%$ & $20 \%$ & $40 \%$ & $60 \%$ & $\mathrm{CV}(\%)$ \\
\hline & \multicolumn{5}{|c|}{1 a 28 dias } \\
\hline $\mathrm{N}$ & 96 & 96 & 96 & 96 & 0,00 \\
\hline $\mathrm{Pl}(\mathrm{g})$ & 38 & 38 & 38 & 38 & 1,70 \\
\hline$P F(g)$ & $495^{b}$ & $520^{a b}$ & $528^{a}$ & $541^{a}$ & 2,98 \\
\hline $\mathrm{GP}(\mathrm{g})$ & $458^{b}$ & $483^{a b}$ & $491^{\mathrm{a}}$ & $505^{a}$ & 3,08 \\
\hline CR(g/ave/dia) & 26 & 27 & 28 & 27 & 6,04 \\
\hline CA & 1,59 & 1,57 & 1,57 & 1,48 & 4,86 \\
\hline VB(\%) & \multicolumn{5}{|c|}{29 a 56 dias } \\
\hline $\mathrm{N}$ & 92 & 92 & 92 & 92 & 0,00 \\
\hline $\mathrm{Pl}(\mathrm{g})$ & 512 & 511 & 513 & 513 & 1,34 \\
\hline PF(g) & 1573 & 1517 & 1534 & 1517 & 2,57 \\
\hline $\mathrm{GP}(\mathrm{g})$ & 1108 & 1052 & 1067 & 1066 & 3,35 \\
\hline CR(g/ave/dia) & ) 94 & 95 & 88 & 84 & 8,01 \\
\hline CA & 2,37 & 2,52 & 2,32 & 2,21 & 6,92 \\
\hline VB(\%) & \multicolumn{5}{|c|}{57 a 84 dias } \\
\hline $\mathrm{N}$ & 88 & 88 & 88 & 88 & 0,00 \\
\hline $\mathrm{Pl}(\mathrm{g})$ & 1485 & 1490 & 1475 & 1478 & 0,51 \\
\hline$P F(g)$ & 2796 & 2755 & 2728 & 2708 & 2,27 \\
\hline $\mathrm{GP}(\mathrm{g})$ & 1309 & 1294 & 1271 & 1230 & 5,55 \\
\hline CR(g/ave/dia) & 151 & 143 & 140 & 136 & 4,85 \\
\hline CA & 3,46 & 3,28 & 3,22 & 3,12 & 4,86 \\
\hline VB(\%) & 100,00 & 99,98 & 99,99 & 100,00 & 0,02 \\
\hline
\end{tabular}

$\mathrm{N}$ : número de aves em cada tratamento; $\mathrm{PI}$ : peso corporal inicial; PF: peso corporal final; GP: ganho de peso; CR: consumo de ração; CA: conversão alimentar; VB: viabilidade; CV: coeficiente de variação; a,b médias seguidas de letras minúsculas distintas na linha diferem estatisticamente pelo teste de Tukey $(p<0,05)$.

dias foram $30,32 \pm 3,88^{\circ} \mathrm{Ce} 22,50 \pm 3,47^{\circ} \mathrm{C}$, respectivamente.

O PF e o GP foram influenciados $(\mathrm{p}<0,05)$ pela inclusão de FRIM nas dietas somente na fase inicial de criação (1 a 28 dias de idade) (tabela II). Observou-se que os valo- 
res de PF e GP aumentaram linearmente $(\mathrm{p}<0,05)$ à medida que se aumentou o nível de inclusão de FRIM na ração dos frangos de corte tipo caipira $\left(\mathrm{PF}=499,17+0,732 \mathrm{x}, \mathrm{r}^{2}=\right.$ 0,$\left.95 ; \mathrm{GP}=46,57+0,748 \mathrm{x}, \mathrm{r}^{2}=0,95\right)$ (figure 1). O CR não foi afetado $(\mathrm{p}>0,05)$ pelos diferentes níveis de FRIM nas rações nas três fases de criação. Apesar da ausência de diferenças significativas entre os tratamentos, foi observada redução na quantidade diária de ração consumida por ave à medida que houve o aumento do nível de FRIM na ração. A análise de variância não detectou diferenças $(p>0,05)$ para CA e VB nas diferentes fases de criação estudadas (tabela II). Não houve diferença $(\mathrm{p}>0,05)$ entre os tratamentos para as variáveis de rendimento de carcaça e de cortes dos frangos de corte tipo caipira aos 84 dias de idade, exceto para o rendimento de cabeça+pescoço (tabela III). Os valores de RGAB dos frangos de corte tipo caipira não foram diferentes $(p>0,05)$ quando as aves foram submetidas a dietas com diferentes níveis de inclusão de FRIM.

Os valores para $\mathrm{pH}, \mathrm{CRA}$ e PPC não foram influenciados ( $\mathrm{p}>0,05)$ pelos níveis de inclusão de FRIM nas dietas de frangos de corte tipo caipira (tabela III). Os níveis de FRIM proporcionaram diferença significativa $(p<0,05)$ sobre a CPPT e a CPCN (tabela III), demonstrando que a utilização do FRIM nas dietas de frangos de corte tipo caipira promoveu uma redução linear $(\mathrm{p}<0,05)$ na coloração das peles da perna e do peito das aves com o aumento do nível de inclusão

Tabela III. Rendimentos de carcaça, de partes e propriedades funcionais da carne de frango de corte tipo caipira submetidos a dietas contendo farelo da raiz integral de mandioca (FRIM) de 56 a 84 dias de idade. (Yield of carcass, parts and meat functional properties of free range broiler chickens fed diets containing cassava root meal (FRIM) from 56 to 84 days old).

Níveis de FRIM

\begin{tabular}{lccccc}
\hline Variáveis & $0 \%$ & $20 \%$ & $40 \%$ & $60 \%$ & $\mathrm{CV}(\%)$ \\
$N$ & 8 & 8 & 8 & 8 & 0,00 \\
RCAR (\%) & 67,60 & 66,93 & 66,09 & 66,61 & 1,58 \\
RPTO (\%) & 27,45 & 26,74 & 27,25 & 27,67 & 4,16 \\
RCSX (\%) & 32,37 & 32,67 & 32,98 & 33,11 & 2,94 \\
RASA (\%) & 12,72 & 12,90 & 13,13 & 12,78 & 3,97 \\
RDOR (\%) & 26,14 & 26,94 & 26,28 & 25,97 & 4,36 \\
RCPC (\%) & $8,78^{\mathrm{b}}$ & $9,26^{\mathrm{ab}}$ & $9,54^{\mathrm{a}}$ & $9,38^{\mathrm{a}}$ & 2,47 \\
RPES (\%) & 6,21 & 6,14 & 6,12 & 6,45 & 5,76 \\
RGAB (\%) & 4,11 & 4,20 & 3,86 & 3,54 & 31,91 \\
pH & 5,78 & 5,82 & 5,73 & 5,77 & 2,07 \\
CRA (\%) & 28,97 & 28,02 & 32,39 & 24,68 & 12,39 \\
PPC (\%) & 18,62 & 17,20 & 17,14 & 17,98 & 28,47 \\
CPCN & $104,84^{\mathrm{a}}$ & $104,08^{\mathrm{ab}}$ & $103,17^{\mathrm{bc}}$ & $102,25^{\mathrm{c}}$ & 0,49 \\
CPPT & $103,25^{\mathrm{a}}$ & $102,67^{\mathrm{a}}$ & $101,92^{\mathrm{b}}$ & $101,17^{\mathrm{c}}$ & 0,33 \\
\hline
\end{tabular}

RCAR: rendimento de carcaça; RPTO: rendimento de peito; RCSX: rendimento de coxa+sobrecoxa; RASA: rendimento de asa; RDOR: rendimento de dorso; RCPC: rendimento de cabeça+pescoço; RPES: rendimento de pés; RGAB: rendimento de gordura abdominal; pH: potencial hidrogeniônico; CRA: capacidade de retenção de água; PPC: perda de peso por cozimento; CPCN: cor de pele de canela; CPPT: cor de pele de peito; CV: coeficiente de variação; a,bmédias seguidas de letras minúsculas distintas na linha diferem estatisticamente pelo teste de Tukey $(p<0,05)$.

Archivos de zootecnia vol. 60, núm. 231, p. 494. 


\section{MANDIOCA PARA FRANGOS DE CORTE TIPO CAIPIRA}

desse ingrediente nas rações (CPPT= 103,298-0,349x, $\mathrm{r}^{2}=0,99$; $\mathrm{CPCN}=104,885+$ $\left.0,434 x, r^{2}=0,99\right)$.

\section{DISCUSSÃO}

Os valores para PF e GP na primeira fase de criação foram obtidos provavelmente devido às dietas com os maiores níveis de FRIM conterem óleo vegetal em elevada proporção, principalmente naquela com $60 \%$ de inclusão de FRIM. O óleo de soja foi acrescentado para suprir a deficiência energética ocasionada pela retirada de grande quantidade de milho nas rações com níveis superiores de FRIM.

A presença de fontes lipídicas nas rações proporciona um menor incremento calórico (IC) o qual representa toda perda de energia durante os processos de digestão, absorção e metabolismo dos nutrientes (Sakomura e Rostagno, 2007). Conseqüentemente, por essa razão, aumentam-se os níveis de energia líquida (EL) metabolizados, uma vez que essa é resultado da energia metabolizável menos a energia perdida como IC, podendo promover melhores resultados de desempenho das aves, visto que influencia diretamente na energia retida como produção animal (Penz Jr. et al., 1999). Em contrapartida, Nascimento etal. (2005) verificaram um efeito quadrático para o GP e relataram que o melhor nível de raspa de mandioca na ração, que éum alimento de composição semelhante ao FRIM, foi de 8,4\%. Os mesmos autores verificaram que o aumento da participação desse ingrediente na dieta de frangos de corte promoveu um decréscimo para o GP nos períodos de 22 a 35 e 36 a 42 dias de idade.

Rostagno et al. (2005) recomendam utilizar níveis entre 5 e $20 \%$ de inclusão de raspa integral de mandioca nas rações de fra1m a inclusão de 40 ou 60\% de FRIM nas dietas, o desempenho foi superior (1 a 28 dias de idade) ou semelhante (29 a 56 e 57 a 84 dias de idade) aos níveis de inclusão recomendados por Rostagno et al. (2005) ou à ração que não continha FRIM.

Embora com valores inferiores, os resultados corroboraram com os encontrados por Gadelha et al. (2006) que verificaram que a farinha integral de mandioca pode ser incluída nas dietas de frangos caipiras na fase de 35 a 84 dias em até $53 \%$ sem prejudicar o desempenho, desde que ocorra um equilíbrio do teor aminoacídico da ração. Da mesma forma, Bezerra et al. (2007) relataram que a substituição do milho pela mandioca não afetou o GP até a idade de 60 dias, porém após esse período observaram uma redução linear nos valores para essa variável.

A diminuição no CR ocorreu, possivelmente, devido as rações com maior nível de FRIM apresentarem uma menor densidade, fazendo com que o papo da ave fosse preenchido por um peso absoluto de ração menor do que a ração que continha basicamente milho e farelo de soja (limitação física). Aves de linhagens geneticamente melhoradas parecem limitar o consumo de ração, mais por uma ação física de enchimento do trato gastrintestinal e da palatabilidade do alimento, do que pela manutenção de peso e composição corporal (Gonzáles, 2002).

Gadelha et al. (2006) não encontraram diferenças significativas para o CR quando incluíram 0, 18, 36 e 53\% de farinha de mandioca nas dietas de frangos de crescimento lento embora também observaram uma menor densidade específica das rações quando as mesmas continham farinha integral de mandioca.

Da mesma forma, Bezerra et al. (2007) verificaram que a CA não foi influenciada pela utilização da raspa de mandioca nos níveis de 0,15 , 30 e 45\% em substituição ao milho das rações de frangos caipiras Label Rouge criados até os 90 dias, sendo obtidos os valores de 3,6; 3,6; 3,7 e 3,9 respectivamente. Também Gadelha et al. (2006) não observaram efeito da inclusão de $0,18,36$ e $53 \%$ de farinha integral de mandioca em dietas de frangos de crescimento lento de 35 a 84 dias de idade, sobre a CA, sendo obser- 
vados os respectivos valores de 2,80; 2,81; 2,92 e 2,88. Ao contrário, Nascimento et al. (2005), relataram resultados indesejáveis para CA, que variaram de 2 a 2,5, à medida que os percentuais de inclusão de raspa de mandioca na ração foram maiores que $10,29 \%$.

Os resultados estão de acordo com Santos et al. (2005) que não encontraram diferenças para os rendimentos de carcaça e de parte de frangos pescoço pelado quando comparados à linhagem paraíso pedrês, no entanto, verificaram que os frangos pescoço pelado obtiveram menor rendimento de cabeça+pescoço em relação ao paraíso pedrês.

Ribeiro et al. (2006) não verificaram diferenças para RCAR e para a maioria dos rendimentos de cortes de frangos de corte tipo caipira aos 84 dias submetidos a dietas contendo 0, 15, 30 e 45\% de FRIM. Embora esses autores tenham encontrado valores superiores de RCAR (78,58; 78,38; 78,73 e $78,38 \%)$ para os respectivos tratamentos, apresentaram RPTO inferiores (26,22; 25,05; 25,70 e 26,18\%). Em contrapartida, Costa et al. (2004) observaram efeito linear para os rendimentos de carcaça e de peito mostrando que à medida que se aumentou o nível de inclusão de raspa de mandioca $(0,5,10,15$, 20 e $25 \%$ de adição deste ingrediente) foram encontrados os menores valores para os parâmetros avaliados.

Os resultados (RGAB) contrastam aos obtidos por Ribeiro et al. (2006) e Costa et al. (2004) que verificaram uma redução no percentual de gordura abdominal com o aumento de FRIM na dieta. Embora não tenham ocorrido diferenças significativas em relação ao RGAB no presente estudo, nota-se que a adição de FRIM às rações foi favorável à redução da gordura. Tendo em vista que, um dos agravantes da criação das linhagens tipo caipira é o maior acúmulo de gordura na carcaça devido a uma maior idade de abate (Kessler et al., 2000), o FRIM, como constituinte das rações, pode ser uma alternativa para solucionar tal problema.

A taxa e o grau de redução do $\mathrm{pH}$ durante a instalação do rigor mortis e conseqüentemente o teor de proteína desnaturada influenciam na CRA e PPC. Se as proteínas não estão desnaturadas, elas continuam a ligar água na conversão do músculo em carne e essa umidade é importante para o rendimento e a qualidade final do produto, contribuindo para a textura, suculência, sabor e palatabilidade da carne como alimento

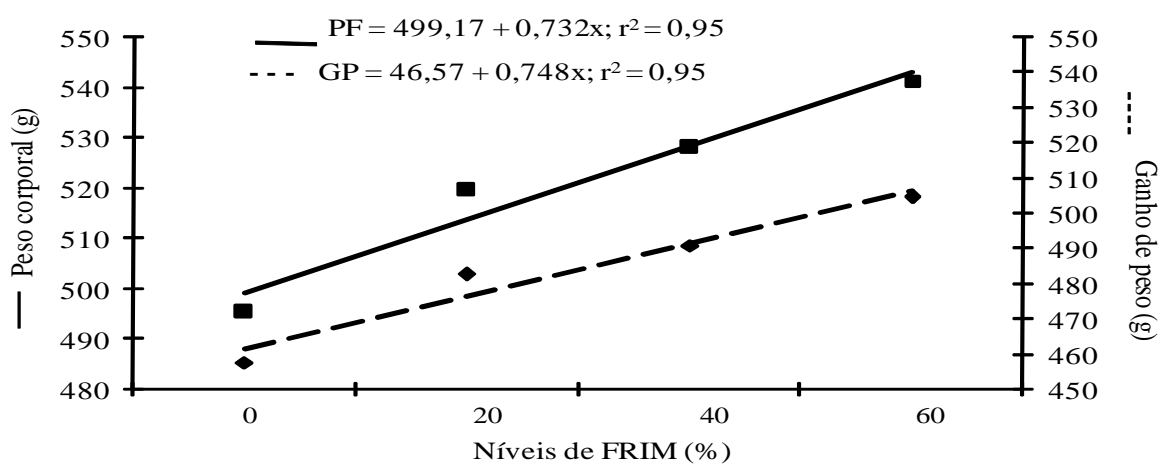

Figura 1. Peso corporal final (PF) e ganho de peso (GP) de frangos de corte tipo caipira submetidos à dietas com diferentes níveis de farelo da raiz integral de mandioca (FRIM) no período de 1 a 28 dias de idade. (Body weight (PF) and weight gain (GP) of free-range broiler chickens submitted diets containing casaba root meal (FRIM) from 1 to 28 days of age).

Archivos de zootecnia vol. 60, núm. 231, p. 496. 


\section{MANDIOCA PARAFRANGOSDE CORTE TIPO CAIPIRA}

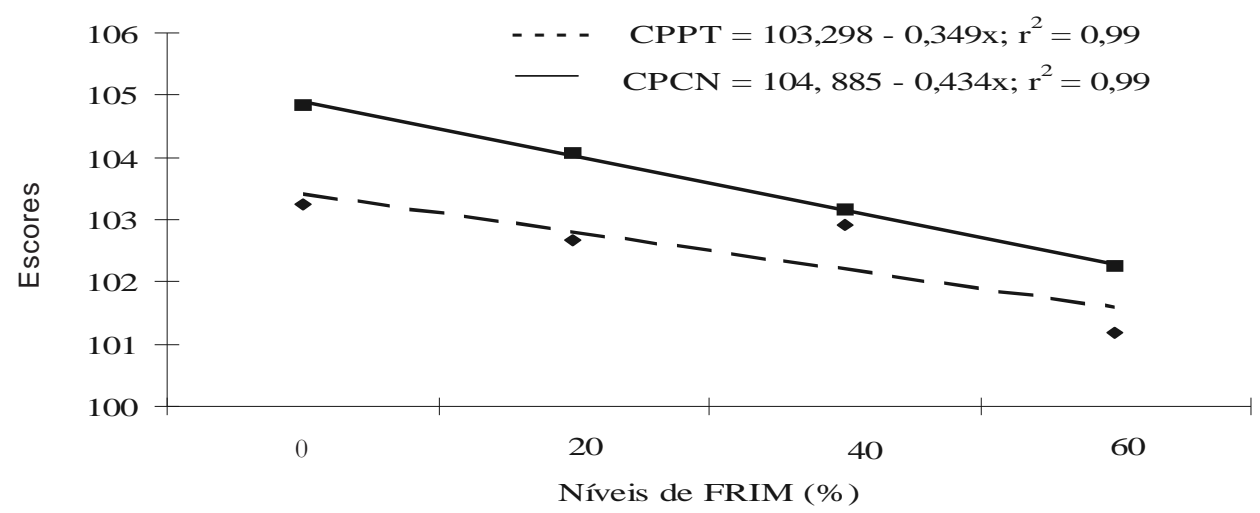

Figura 2. Cor de pele de peito (CPPT) e cor de pele de canela (CPCN) de frangos de corte tipo caipira submetidos a dietas contendo farelo da raiz integral de mandioca (FRIM) de 56 a 84 dias. (Breast skin colour (CPPT) and shank skin colour (CPCN) of fre-range broiler chickens submitted diets containing casaba root meal (FRIM) from 56 to 84 days of age).

(Olivo e Shimokomaki, 2006).

Os resultados para $\mathrm{pH}$ apresentados na tabela III foram muito próximos aos observados por Santos et al. (2005) para frangos de corte machos da linhagem ISA label $(\mathrm{pH}=$ $5,69)$ e do valor considerado normal $(\mathrm{pH}=$ 5,68 ) para carne de ave 24 horas post mortem (Schneider et al., 2006). Fanatico et al. (2005) verificaram que carne de aves de crescimento lento criadas em sistema semi-intensivo apresentaram maior perda de exsudato e perda no cozimento $(\mathrm{p}<0,05)$ do que carne de aves de crescimento rápido semi-confinadas, possivelmente pelo fato de que aves de crescimento lento possuem, à mesma idade, menor espessura de carne de peito e também maior umidade de carne.

Aves mantidas em piquetes de gramíneas consomem grande quantidade de xantofilas (derivado de $\beta$-caroteno) presentes nas plantas verdes e também no milho, esses pigmentos são transferidos para a pele, pernas e bico dos frangos de corte (Leeson e Summers, 2001). No entanto no presente estudo, os níveis de FRIM proporcionaram diferença significativa $(\mathrm{p}<0,05)$ sobre a CPPT e a CPCN (tabela III), demonstrando que a utilização do FRIM nas dietas de frangos de corte tipo caipira promoveu uma redução linear $(\mathrm{p}<0,05)$ na coloração das peles da perna e do peito das aves com o aumento do nível de inclusão desse ingrediente nas rações (figura 2).

Freitas et al. (2007), avaliando a pigmentação das canelas de frangos de corte, mostraram uma diminuição linear com o acréscimo de $30 \%$ de farinha de varredura de mandioca nas dietas e alegaram que a utilização de determinados alimentos alternativos substituindo o milho nas rações de frangos de corte pode prejudicar a coloração da carcaça e canelas das aves. Os resultados observados devem ter ocorrido provavelmente devido à quantidade de $\beta$-caroteno da mandioca $(0,01 \mathrm{mg} / \mathrm{kg})$ (Cereda, 2001) ter sido insuficiente para promover a coloração mais intensa nas peles de peito e perna dos frangos caipira, visto que segundo FAO (2007), o milho possui $125 \mathrm{mg} / \mathrm{kg}$ de $\beta$-caroteno. Os consumidores associam normalmente as colorações amareladas de pele e canela a produtos originados de criações caracterizadas como mais próximas do natural (Leeson e Summers, 2001) e a coloração mais clara da pele dos frangos de corte tipo caipira submetidos a dietas contendo elevados níveis de FRIM poderia dificultar a aceitação 
das carcaças pelo mercado.

\section{CONCLUSÕES}

O farelo da raiz integral de mandioca pode ser utilizado nas dietas de frangos de corte tipo caipira na proporção de $60 \%$ da ração total sem prejudicar o desempenho e os rendimentos de carcaça e de cortes das

\section{BIBLIOGRAFIA}

Bezerra, A.V., Rodrigues, K.F., Silva, G.F., Sousa, J.P.L., Santos, P.M., e Caraça, D.C. 2007. Substituição do milho pelo resíduo da fabricação da fécula da mandioca em rações de frangos de crescimento lento label rouge. Em: Congresso Latinamericano de Avicultura, 20. Anais... FACTA. Porto Alegre. pp. 92-94.

Carrijo, A.S., Menezes, G.P., Silva, M.J., Oliveira, M.S.S, e Onselen, V.J. 2002. Utilização do farelo de raiz de mandioca como fonte energética alternativa na engorda de frango tipo caipira. Em: Reunião Anual da Sociedade Brasileira de Zootecnia, 39. Anais... Sociedade Brasileira de Zootecnia. Recife.

Cereda, M.P. 2001. Caracterização dos subprodutos da industrialização da mandioca. Em: Culturas de tuberosas amiláceas latinoamericanas: Manejo, uso e tratamento de subprodutos da industrialização da mandioca. Fundação Cargill. São Paulo. pp. 11-37.

Costa, F.G.P., Nascimento, G.A.J, Barros, L.R., Amarante Jr., V.S., Brandão, P.A., e Pereira, W.E. 2004. Avaliação de carcaça de frangos de corte aos 49 dias de idade, submetidos a diferentes níveis de raspa de mandioca em substituição ao milho. Rev. Bras. Ciênc. Avíc., 6: 86.

Enriquez, F.Q. and Ross, E. 1967. The value of cassava root meal for chicks. Poultry Sci., 46: 622-626.

ESTAT 2.0. s.d. Sistema de análise estatística. Pólo Computacional-Departamento de Ciências Exatas-UNESP. Jaboticabal.

Fanatico, A.C., Cavitt, L.C., Pillai, P.B., Emmert, J.L., and Owens, C.M. 2005. Evaluation of slowergrowing broiler genotypes grown with and without outdoor access: meat quality. Poultry Sci., 84: 1785-1790. aves. As propriedades funcionais da carne de frangos de corte tipo caipira não são alteradas pela inclusão do farelo da raiz integral de mandioca.

O farelo da raiz integral de mandioca não promove coloração adequada das peles das canelas e do peito de frangos de corte tipo caipira.

FAO. 2007. Composição aproximativa dos alimentos. http://www.fao.org/docrep/007/x3996p/ x3996p18.htm. (21/12/07).

Freitas, C.R.G., Ludke, M.C.M.M., Ludke, J.V., Rabello, C.B.V., Nascimento, G.R., e Torres, T.R. 2007. Efeito da inclusão da farinha de varredura de mandioca em dietas de frangos de corte sobre as características de carcaça. Em: Reunião Anual da Sociedade Brasileira de Zootecnia, 44. Anais... Sociedade Brasileira de Zootecnia. Jaboticabal.

Gadelha, A.C., Rolim, B.N., Santos, E.J.F., Alvite, E.C., Pinto, M.J.F.D., Pinto, J.L.B. e Souza, F.M. 2006. Farinha integral de mandioca em rações para frangos de crescimento lento de 35 a 84 dias de idade. Em: Reunião Anual da Sociedade Brasileira de Zootecnia, 43. Anais... Sociedade Brasileira de Zootecnia. João Pessoa.

Garcia, M.and Dale, N. 1999. Cassava root meal for poultry. J. Appl. Poultry Res., 8: 132-137.

Gonzales, E. 2002. Ingestão de alimentos: mecanismos regulatórios. In: Fisiologia aviária aplicada a frangos de corte. FUNEP/UNESP. Jaboticabal. 375 pp.

Hellmeister Filho, P. 2002. Efeitos de fatores genéticos e do sistema de criação sobre o desempenho e o rendimento de carcaça de frangos tipo caipira. ESALQ/USP. Piracicaba.

Kessler, A.M., Snizek, P.N. e Brugalli, I. 2000. Manipulação da quantidade de gordura na carcaça de frangos. Em: Conferencia Apinco de Ciencia e Tecnologia Avícola. Anais... FACTA. Campinas. pp. 107-133.

Lesson, S. and Summers, J.D. 2001. Non-nutritive feed addititives. In: Nutrition of the chicken. $4^{\text {a }}$ ed. University Books. Ontario. 6: 429-455.

Nascimento, G.A.J., Costa, F.G.P., Amarante Jr, V.S. e Barros, L.R. 2005. Efeitos da substituição

Archivos de zootecnia vol. 60, núm. 231, p. 498. 


\section{MANDIOCA PARA FRANGOS DE CORTE TIPO CAIPIRA}

do milho pela raspa de mandioca na alimentação de frangos de corte durante as fases de engorda e final. Ciênc. Agrotec., 29: 200-207.

Olivo, R. e Shimokomaki, M. 2006. Fatores que influenciam as características das matériasprimas e suas implicações tecnológicas. Em: Atualidades em ciência e tecnologia de carnes. Varela. São Paulo. pp. 17-25.

Penz Jr., A.M.; Kessler, A.M. e Brugalli, I. 1999. Novos conceitos de energia para aves. Em: Simpósio internacinal sobre nutricão de aves. Anais... FACTA-Fundação APINCO de Ciência e Tecnologia Avícolas. Campinas. pp. 1-20.

Ribeiro, S.S., Carrijo, A.S., Sanches, J.F., Fascina, V.B., Maruyama, L.M.N. e Higa, J.A. 2006. Rendimento de carcaça e cortes de frangos tipo caipira alimentados com farelo de raiz integral de mandioca. Rev. Bras. Ciênc. Avíc., 8: 161. Rostagno, H.S., Albino, L.F.T., Donzele, J.L., Gomes, P.C., Oliveira, R.F., Lopes, D.C., Ferreira, A.S. e Barreto, S.L.T. 2005. Tabelas brasileiras para aves e suínos: composição de alimentos e exigências nutricionais. $2^{\mathrm{a}}$ ed. UFV. Viçosa. $186 \mathrm{pp}$.

Sakomura, N.K. e Rostagno, H.S. 2007. Métodos de pesquisa em nutrição de monogástricos. FUNEP. Jaboticabal. 283 pp.

Santos, A.L., Sakomura, N.K., Freitas, E.R., Fortes, C.M.S. and Carrilho, E.N.V.M. 2005. Comparasion of free range broiler chicken strains raised in confined or semi-confined systems. Braz. J. Poultry Sci., 7: 85-92.

Scheinider, J.P., Oda, S.H.I e Guarnieri, P.D. 2006. Carne DFD em frangos. Em: Atualidades em ciência e tecnologia de carnes. Varela. São Paulo. pp. 105-113.

Takahashi, S.E., Mendes, A.A., Saldanha, E.S.P.B., Pizzolante, C.C., Pelícia, K., Garcia, R.G., Paz, I.C.L.A. e Quinteiro, R.R. 2006. Efeito do sistema de criação sobre o desempenho e rendimento de carcaça de frangos de corte tipo colonial. Arq. Bras. Méd. Vet. Zootec., 58: 624-632.

UBA. 2007. Desempenho do frango de corte. http:// www.uba.org.br. (21/12/07). 